\title{
Xenotransplantation
}

Official Journal of the International Xenotransplantation Association

\section{Rotational culture and integration with amniotic stem cells reduce porcine islet immunoreactivity in vitro and slow xeno-rejection in a murine model of islet transplantation.}

\begin{tabular}{|c|c|}
\hline Journal: & Xenotransplantation \\
\hline Manuscript ID & XEN-18-0-0093 \\
\hline Manuscript Type: & Original Article \\
\hline Date Submitted by the Author: & 12-Sep-2018 \\
\hline Complete List of Authors: & $\begin{array}{l}\text { Zafar, Ali; The Islet Research Laboratory, Worcestershire Acute Hospitals } \\
\text { NHS Trust } \\
\text { Lee, Jou; The Islet Research Laboratory, Worcestershire Acute Hospitals } \\
\text { NHS Trust } \\
\text { Yesmin, Shameema; The Islet Research Laboratory, Worcestershire Acute } \\
\text { Hospitals NHS Trust } \\
\text { Paget, Michelle; The Islet Research Laboratory, Worcestershire Acute } \\
\text { Hospitals NHS Trust } \\
\text { Bailey, Clifford; Diabetes Research, Aston University } \\
\text { Murray, Hilary; The Islet Research Laboratory, Worcestershire Acute } \\
\text { Hospitals NHS Trust } \\
\text { Downing, Richard; The Islet Research Laboratory, Worcestershire Acute } \\
\text { Hospitals NHS Trust }\end{array}$ \\
\hline Keywords: & $\begin{array}{l}\text { porcine islets, xeno-transplantation, T-cell, amniotic epithelial cells, } \\
\text { rotational cell culture, immunomodulation }\end{array}$ \\
\hline Topics: & Cells - Islets, Cells - Stem cells \\
\hline
\end{tabular}


2

3

4

5

6

7

8

\begin{abstract}
Background

Pre-transplant modification of porcine islets may improve their suitability for clinical use in diabetes management by supporting graft function and reducing the potential for xenorejection. The present study investigates intra-graft incorporation of stem cells that secrete beta $(\beta)$-cell trophic and immunomodulatory factors to preserve function and alter immune cell responsiveness to porcine islets.
\end{abstract}

\title{
Methods
}

Isolated porcine islets were maintained in a 3-dimensional rotational cell culture system (RCCS) to facilitate aggregation with human amniotic epithelial cells (AEC). Assembled islet constructs were assessed for functional integrity and ability to avoid xeno-recognition by CD4+ T-cells using mixed islet:lymphocyte reaction assays. To determine whether stem cellmediated modification of porcine islets provided a survival advantage over native islets, structural integrity was examined in a pig-to-mouse islet transplant model.

\section{Results}

RCCS supported the formation of porcine islet:AEC aggregates with improved insulinsecretory capacity compared to unmodified islets, whilst the xeno-response of purified CD4+ T-cells to AEC-bearing grafts was significantly $(\mathrm{p}<0.05)$ attenuated. Transplanted AECbearing grafts demonstrated slower rejection in immune-competent recipients compared to unmodified islets. 


\section{Conclusions/interpretation}

Rotational culture enables pre-transplant modification of porcine islets by integration with immunomodulatory stem cells capable of subduing xeno-reactivity to CD4+ T-cells. This reduces islet rejection and offers translational potential to widen availability and improve the clinical effectiveness of islet transplantation.

Keywords: porcine islets, xeno-transplantation, T-cell, amniotic epithelial cells, rotational cell culture, immunomodulation
Abbreviations
AEC - amniotic epithelial cells
ASC - amniotic stem cells
HARV - high aspect ratio vessel
IEQ - islet equivalent
MILR - mixed islet lymphocyte reaction
RCCS - rotational cell culture system 


\section{INTRODUCTION}

Effective and sustainable glucose homeostasis with islet replacement therapy in insulindependent diabetes requires both adequate mass and functional integrity of implanted beta ( $\beta$ )-cells. Early loss of the structural and functional viability of transplanted islets due to the condition of the donor pancreas or the isolation process is likely to result in acute rejection, restricted engraftment and/or poor graft performance ${ }^{1,2}$. In the longer term, the vulnerability of islets to recurrent auto-immune destruction and/or allo-rejection leads to $\beta$-cell attrition and loss of glucose regulation ${ }^{3,4}$.

A period of pre-transplant islet culture allows time for $\beta$-cell recovery. Conventional static culture systems do not adequately preserve islet viability prior to transplantation ${ }^{5,6}$, but we previously demonstrated that a 3-D rotational cell culture system (RCCS) provides a more suitable environment, supporting integrity of human islets for up to 10 days ${ }^{7}$. The more favourable conditions created within these dynamic 3-D bioreactor culture systems improve oxygenation, nutrient delivery and metabolite removal to preserve tissue viability ${ }^{7}$. The low gravity micro-environment of the RCCS also facilitates pre-transplant tissue regeneration and remodelling. Of note, we have reported that such systems facilitate the co-aggregation of human islets with immunomodulatory stem cells sourced from human amniotic membrane ${ }^{8,9}$. This offers the ability to alter the responsiveness of immune cell populations, especially those with direct involvement in islet graft rejection, whilst proffering trophic support ${ }^{8,9}$. Amnionderived stem cells (ASC) exhibit immune-privilege, most likely via low-level expression of surface MHC class I and II, whilst the factors within the ASC secretome include a range of pro-angiogenic, cell trophic, anti-inflammatory and immunomodulatory cytokines ${ }^{10-12}$.

Despite these potential benefits in the context of clinical islet allo-transplantation, the 
behaviour of human ASC populations for xenogenic immune events has not been widely characterised.

The limited supply of suitable donor pancreases from which to yield transplant-grade islets curtails wider use of $\beta$-cell replacement therapy. However, porcine islet surrogates with a physiology similar to humans are increasingly being considered for clinical application as the risks from porcine endogenous retroviruses can be eliminated ${ }^{13}$. Although xeno-rejection remains a major challenge, various immune-isolation strategies using biomaterials are in development. These include trials to evaluate the clinical efficacy of encapsulated porcine islets ${ }^{14}$, and localised immune-modulation strategies may strengthen the case for use of nonhuman islet donors ${ }^{15,16}$.

This study explores the inherent trophic and immuno-modulatory properties of a distinct population of ASC; human amniotic epithelial cells (AEC). Here we extend previous studies with human islets ${ }^{8,9}$, to determine the ability of AEC to support porcine islet function and alter their CD4+ xeno-reactivity by co-integration into transplantable heterotypic cell constructs using 3D RCCS bioreactor technology. The approach is interrogated in vivo, observing structural integrity of the graft and tempo of xeno-rejection of the heterotypic constructs in a murine model of islet transplantation. 


\section{MATERIALS AND METHODS}

Unless otherwise stated, reagents were obtained from Sigma-Aldrich, Dorset UK.

\subsection{Porcine islet isolation}

Pancreases from market aged pigs were obtained through a local abattoir with the appropriate permissions for the collection, transportation and use of animal by-products for educational and research purposes (Animal Health and Veterinary Laboratories Agency (AHVLA) registration number: C1123407; Animal By-Product (ABP) reference number: U1115466). Ten pancreases with average cold ischemia time 3 hours were processed and islets isolated using a modified version of the manual human islet isolation method previously described ${ }^{17}$. Briefly, pancreases were subjected to enzymatic digestion $(1.5 \mathrm{mg} / \mathrm{ml}$ collagenase XI by ductal infusion) with mechanical agitation, and islets separated from the crude extract by density gradient centrifugation on Ficoll columns. Purified islets were stained with dithizone $(500 \mu \mathrm{g} / \mathrm{ml})$, counted and converted to islet equivalents (IEQ; 1IEQ equivalent to an islet measuring $150 \mu \mathrm{m}$ in diameter). Islet cell viability was confirmed by Trypan Blue $(0.4 \% \mathrm{v} / \mathrm{v})$ exclusion. Islets were re-suspended in Medium 199 (M199) supplemented with 10\% porcine serum, $1 \%$ non-essential amino acids, $3 \mathrm{mM}$ L-glutamine and $10 \mu \mathrm{M}$ nicotinamide (vitamin B3) with anti-microbial agents; $100 \mathrm{U} / \mathrm{ml}$ penicillin, $100 \mu \mathrm{g} / \mathrm{ml}$ streptomycin, $10 \mu \mathrm{g} / \mathrm{ml}$ amphotericin B until use in the 3-D culture studies.

\subsection{Human Amniotic Epithelial Cell (AEC) isolation}

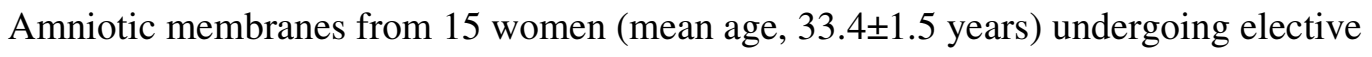
Caesarean section at term were obtained with ethical approval and informed consent. Amniotic epithelial cells (AEC) were isolated according to existing protocols ${ }^{8}$. Briefly, sections of amnion were separated from the chorion layer by blunt dissection, rinsed in 
phosphate buffered saline (PBS), minced and digested in $0.25 \%$ (w/v) trypsin in Hanks balanced salt solution (HBSS) for 20 minutes at $37^{\circ} \mathrm{C}$. The resulting tissue suspension was filtered through a $500 \mu \mathrm{m}$ mesh, retaining larger pieces of amnion for further digestion with trypsin, and liberating all available epithelial cells. The AEC suspension was centrifuged, resuspended in RPMI 1640 supplemented with 10\% fetal bovine serum (FBS), 100U/ml penicillin, $100 \mu \mathrm{g} / \mathrm{ml}$ streptomycin and $10 \mu \mathrm{g} / \mathrm{ml}$ amphotericin B and seeded at a density of $2.5 \times 10^{5} / \mathrm{ml}$ in $\mathrm{T}-75$ flasks, cultured at $37^{\circ} \mathrm{C}$ in an atmosphere of $5 \% \mathrm{CO}_{2}$ for 72 hours to allow adherence followed by a $50 \%$ medium change. AEC monolayers reached confluence within 7 days and were used at passage (P) 1 .

\subsection{Rotational cell culture of porcine islets: integration with human AEC}

To determine if the RCCS would support extensive porcine islet modification without compromising $\beta$-cell viability or function, extended (viz. $>24$ hour), islet culture was conducted using a Synthecon RCCS as previously described ${ }^{7,8,18}$. Porcine islets in suspension were seeded at 500-1000 IEQ per $\mathrm{ml}$ into 10ml high aspect ratio vessels (HARV, Cellon Ltd, Bereldange, Luxembourg) in supplemented M199. For co-culture studies, confluent AEC monolayers at P1 were disrupted by mild enzymatic digestion $(0.025 \%$ trypsin-EDTA), the cell suspension washed in PBS and added to the islet cultures at a final density of $1 \times 10^{5}$ cells per ml. Porcine islet monocultures and islet:AEC co-cultures were maintained under rotating, low gravity conditions ${ }^{7,9,18}$ for 48 hours at $37^{\circ} \mathrm{C}$ and $5 \% \mathrm{CO}_{2}$, prior to morphological and functional assessment.

\subsection{Construct assessment: histology}

Cell constructs formed by rotational culture of porcine islets in the presence or absence of AEC were assessed under light microscopy using Trypan Blue to assess integrity and 
dithizone staining to identify and determine the interaction of $\beta$-cells both with each other and non $\beta$-cell populations. For immunocytochemical analysis the cellular constructs, consisting of islet (endocrine) cells alone or islets associated with AEC, were fixed with 4\% paraformaldehyde (w/v) for 30 mins at room temperature (RT). Washes in PBS were followed by permeabilisation $(0.3 \%$ Triton-X-100), blocking with $10 \%$ normal goat serum in PBS (Vector Laboratories Ltd, Peterborough, UK) and incubation with primary antibodies, anti-human cytokeratin 19 (CK19; Dako UK Ltd, Cambridgeshire, UK- 1:100) and antihuman insulin (Abcam, Cambridge, UK 1:50) for 1 hour at RT and $4^{\circ} \mathrm{C}$ overnight. Secondary antibody (goat anti-mouse IgG-FITC for CK19 and goat anti-rabbit IgG- TRITC for insulin - Cambridge Biosciences, Cambridge, UK, 1:100) was applied for 3 hours at RT. Processed cells were rinsed, mounted in fluorescence mounting medium and imaged using a Zeiss Axioskop 40 fluorescence microscope and AxioCam MRc colour camera (Carl Zeiss, Hertfordshire, UK).

\subsection{Construct assessment - secretory capacity}

The impact on insulin secretory capacity resulting from porcine islet integration with AEC was assessed following 48 hours within the RCCS and compared to unmodified porcine islets maintained under the same culture conditions. Static challenge assays determined insulin secretion under basal conditions $(1.67 \mathrm{mmol} / \mathrm{l}$ glucose in HBSS), and subsequent to stimulation with high glucose $(16.7 \mathrm{mmol} / \mathrm{l})$ supplemented with arginine $(10 \mathrm{mmol} / \mathrm{l})$ or the aforementioned insulin secretagogues combined with $10 \mathrm{mmol} / \mathrm{l}$ theophylline. The assays were performed as previously described ${ }^{7,8,18}$ employing 1 hour incubation periods. Stimulated insulin release expressed as a ratio of insulin secretion under basal conditions (Stimulation Index, S.I.) is an indicator of $\beta$-cell viability and forms part of the pre-transplant islet assessment in the clinical islet transplant protocol ${ }^{19}$. Stimulation index was measured in 
preference to total insulin output per islet (or IEQ) to reflect the changes in overall cellular composition of the constructs formed by culture within the RCCS and integration with AEC. For these studies porcine islets (as IEQ) were co-cultured with AEC in ratios of 1:100, and 1:1000 to determine whether the trophic potential of the AEC on islet secretory capacity was dose-responsive. Hormone output was measured using a commercial ELISA specifically for porcine insulin (Diagenics Ltd, Milton Keynes, UK).

\subsection{In vitro immunomodulation studies}

\subsubsection{Human peripheral blood mononuclear cell isolation; enrichment of CD4+ T-cells}

Peripheral blood mononuclear cells (PBMC) were isolated from leucocyte concentrates (CD leucocyte cones) obtained from 12 healthy donors through the National Blood Service (NBS, Birmingham, UK) with local research ethics approval. Platelet depleted PBMC were obtained using the SepMate ${ }^{\mathrm{TM}}$ cell purification system (StemCell Technologies, Cambridge, UK) and CD4+ T-cell subsets were isolated using a commercial kit (Dynabeads ${ }^{\circledR}$ Untouched $^{\mathrm{TM}} \mathrm{T}$-cell isolation kits, Life Technologies, Paisley, UK) according to manufacturer's instructions. Previous flow cytometry analysis confirmed that the purity of the T-cell populations obtained by this method is greater than $95 \%^{9}$.

\subsubsection{Mixed porcine islet:lymphocyte reaction assays: porcine islets/islet:AEC constructs versus human CD4+ T-cells}

To determine the human T-cell immune-response to unmodified porcine islets and islet-AEC constructs assembled under RCCS conditions, mixed islet:lymphocyte reaction (MILR) assays were performed after 48 hours of 3D culture. Islets or islet:AEC aggregates (in ratios 1:100 and 1:1000) were transferred from the HARVs to 24-well tissue culture-treated plates (50-100 IEQ/well). A 48 hour period of culture in serum-containing medium facilitated firm 


\subsection{In vivo assessment of construct integrity and survival}

\subsubsection{Transplantation}

We sought to determine the impact of RCCS pre-transplant culture and integration with AEC on porcine islet survival in immune competent non-diabetic mice recipients. At this stage the aim was not to show enhanced graft function or reversal of hyperglycaemia but rather that stem cell-mediated modification of the porcine islet graft provided it with a survival advantage over native islets. The study was conducted in accordance with the UK Animal (Scientific Procedures) Act 1986. Eight week old female C57BL/6 mice raised in-house were used as islet graft recipients. Animals were housed and maintained in an air conditioned room at $22 \pm 2 \mathrm{C}$ with a lighting schedule of $12 \mathrm{~h}$ light $(0800-2000 \mathrm{~h})$ and $12 \mathrm{~h}$ dark. A standard pellet diet with tap water was supplied ad libitum. A group of animals $(n=3)$ was pre-treated with immunosuppressants as per the standard islet transplant protocol ${ }^{21}$, a treatment regimen that continued up to the day of graft harvest. Islet implants consisting either of unmodified 
porcine islets (control) or porcine islet:AEC constructs as described above were prepared for transplantation following 48 hours of culture within the RCCS. On the day of the study unmodified islets and islet:AEC constructs (ratio 1:100) were removed from the HARVs, pelleted (400rpm) re-suspended in culture medium, counted and assessed for viability using Trypan Blue exclusion. For implantation cell constructs equating to 1000 IEQ were aspirated into an intravenous cannula (16G, 48mm - NHS Supply Chain, Alfreton, UK) and gently placed either under the kidney capsule or left liver lobe of mice under isoflurane anaesthesia. Control islets were transplanted either in the absence or presence of systemic immunosuppression. Islet:AEC constructs were transplanted in the absence of systemic immunosuppression. Animals were allowed to recover and monitored daily for the first seven days and weekly until explantation of the grafted tissue.

\subsubsection{Ex vivo analysis}

Following euthanasia graft-bearing kidneys and livers were excised and processed for analysis by immunocytochemistry. Organs were fixed whole in paraformaldehyde $(4 \% \mathrm{w} / \mathrm{v})$, embedded in paraffin, and cut into 5- $\mu \mathrm{m}$ sections for processing, haematoxylin and eosin (H\&E) and immune-staining. For immunocytochemical analysis rehydration preceded an antigen retrieval step in Tris-EDTA buffer (pH 9.00) and blocking with 10\% normal goat serum (NGS) (Vector Laboratories) for $1 \mathrm{~h}$. Tissues were stained with an anti-insulin antibody diluted at 1:50 (Abcam) and an anti-CK19 antibody diluted at 1:100 (DAKO) in 5\% NGS. Secondary antibodies used were Alexa Fluor 594 goat anti-rabbit and Alexa Fluor 488 goat anti-mouse (Abcam) diluted at 1:100 in 5\% NGS. Slides were cover slipped with fluorescence mounting medium and visualised using a Zeiss Axioskop 40 fluorescence microscope and AxioCam MRc colour camera (Carl Zeiss). 


\subsection{Data Analysis}

ANOVA and Dunnett's multiple comparison post hoc tests assessed the impact of islet modification on insulin secretory capacity and CD4+ immune-reactivity. A p value $<0.05$ was considered statistically significant, as found using SPSS software version 24 (IBM Corp., Armonk, New York, USA). 


\section{RESULTS}

\subsection{Morphology and function of porcine islets cultured alone or with AEC within the RCCS}

Porcine islets are prone to fragmentation during the isolation process (Fig 1A), but consistent with our previous observations with human islets, the structural integrity of the porcine islets was improved during RCCS culture, as they formed constructs of $150-200 \mu \mathrm{m}$ in diameter over a period of 48 hours (Fig.1B). When the porcine islets were co-cultured with AEC in the RCCS the AEC adhered to the periphery of the islets (Fig.1C-E). However, the AEC did not completely encapsulate the islets as previously noted for human islet-AEC co-culture ${ }^{8}$ (Fig. $1 \mathrm{~F}-\mathrm{H})$.

Glucose challenge assays confirmed that physiologically-appropriate insulin secretion was preserved after 48 hours of RCCS for both the unmodified islets and AEC-bearing islet aggregates (Fig.2 upper panel). Co-culture with AEC was associated with a trophic effect on $\beta$-cell function, particularly after co-culture with high numbers of AEC (1:1000) where the observed insulin secretion yielded an S.I. of $2.19 \pm 0.13$ and $3.02 \pm 0.12$ in response to high glucose + arginine and high glucose + arginine + theophylline respectively as compared to $1.49 \pm 0.10$ and $1.72 \pm 0.13$ respectively as seen with unmodified islets $(p<0.05$ - Fig. 2 upper panel).

\subsection{T-cell allo-response of CD4+ T-cells to AEC-bearing porcine islet constructs assembled and held under rotational cell culture conditions}

As porcine islet xeno-rejection appears to be mediated by the actions of activated CD4+ Tcells $^{22}$, mixed islet:lymphocyte reaction assays were undertaken with AEC-bearing porcine 
islet constructs. Human CD4+ T-cell expansion occurred in response to anti-CD3/CD28 (bead) stimulation and the co-culture of these cells with unmodified porcine islets further enhanced CD4+ proliferation. There was substantial variability in the responses of naïve CD4+ T-cells to the activator anti- CD3/CD28 and to unmodified porcine islets. The variance in responses is likely to reflect the different donor tissues which would influence the matching of human versus porcine leucocyte antigens, the T-cell receptor repertoire and the ability of resident APCs to present antigens ${ }^{23,24}$. Although the magnitude of response of activated CD4+ in the presence or absence of unmodified porcine islets was not significantly different, there was a pronounced $(>60 \%)$ attenuation of CD4+ T-cell expansion with the porcine islet:AEC constructs, with a similar degree of inhibition at the two concentrations of AEC used in the co-cultures ( $\mathrm{p}<0.05$, Fig.2 lower panel). This indicates that human AEC influenced the T-cell response to xenogeneic islets to a similar degree to that previously reported for allogeneic tissue ${ }^{8,9}$.

\subsection{Altered immunoreactivity and tempo of islet xeno-graft rejection of AEC-bearing islets}

Histological analysis of explanted graft tissue suggests an altered tempo of rejection of unmodified islets compared to AEC-bearing islet grafts during the period of study. Fig 3 illustrates islets grafted into the left liver lobe and harvested 14 days post-transplant, showing the location of the original graft site with $\mathrm{H} \& \mathrm{E}$ staining (Fig $3 \mathrm{~A}, \mathrm{C}, \mathrm{E}$ ). Insulin-positive staining cells were clearly present at the graft site in livers of immune-suppressed mice receiving unmodified islets (Fig 3 B), and very low insulin immunoreactivity was measured in grafts of unmodified islets transplanted to non-immune-suppressed mice (Fig 3 D). By comparison, sections of AEC-bearing constructs transplanted into the livers of immunecompetent recipients exhibited sustained graft integrity at 14 days with both CK19 and 
insulin immunoreactivity persisting at the graft site (Fig.3 F). Insulin expression was also observed for grafts into the livers of immunosuppressed recipients at $>30$ days post-transplant but the levels of insulin expression were lower in those receiving the islet-AEC grafts. There was no evidence of longer term graft survival of unmodified islets in immune competent recipients.

To determine the post-transplant spatial arrangement of the islets and AECs which comprise the implanted constructs, immunocytochemical analysis was undertaken on graft material introduced beneath the kidney capsule. The staining showed insulin immunoreactivity in graft bearing kidneys at 7, 14 and >30 days post-transplantation. Insulin and CK19-positive cells remained in close proximity, especially in the 7 day grafts (Fig 4A-D) where co-localisation of the two cell types was widely observed. At $>30$ days post-transplantation there was a marked reduction in graft area and in the numbers of $\beta$-cells and AEC at the graft site (Fig.4E,F). However, by this time no islet material could be found in the kidneys of immune competent mice receiving unmodified islets (data not shown). 


\section{DISCUSSION}

Current strategies to improve $\beta$-cell viability in the peri-transplant period are based largely on chemical or pharmacological interventions to enable cyto-protection during the islet isolation procedure ${ }^{25}$, provide trophic support during pre-transplant culture ${ }^{26}$ or reduce the inflammatory/immune response ${ }^{27}$. Whilst each is individually beneficial to preserve islet integrity, combined multiple pharma-based interventions introduce substantial complexity to the clinical islet transplant protocol. This poses challenges to obtaining regulatory approval and for the standardization of protocols in different islet isolation and transplantation centres. Also, the risk of significant drug-drug interactions and potential longer-term detrimental impact on the graft or the recipient must be considered.

A successful strategy would enhance islet engraftment, suppress rejection and sustain an effective $\beta$-cell mass without adding undue risks. In the present study porcine islets were adopted to overcome the shortage of human islets. To circumvent pharmacological intervention, porcine islets were maintained by 3D rotational cell culture to allow pretransplant islet modification within a more $\beta$-cell supportive environment than static culture, preserving structural viability and nutrient responsiveness as previously demonstrated with human islets ${ }^{7,18}$. The approach is further enhanced by incorporation of human AEC, shown to have localised $\beta$-cell trophic ${ }^{8}$, anti-inflammatory ${ }^{28}$ and immunomodulatory ${ }^{29}$ properties. Notwithstanding the disparity in species of origin, the RCCS co-culture of porcine islets and human AECs resulted in successful physical integration of the two cell types to form constructs with AEC at the islet surface. As previously reported for human islet:AEC constructs ${ }^{8,9}$, RCCS-mediated attachment of AEC to the islet periphery, despite incomplete coverage, is capable of altering porcine islet capacity for sustained function and immunomodulation. 
The cross-species cellular interaction of human AEC and porcine islets had no adverse consequence for $\beta$-cell function, instead deriving benefits from the bioactive components of the AEC secretome. AECs synthesise and secrete growth factors with the potential to sustain islet function with reported mRNA expression of TGF $\beta, \mathrm{EGF}^{30}$ and $\mathrm{KGF}^{31}$ associated with improved islet engraftment and $\beta$-cell proliferation ${ }^{32-34}$. The dose-dependent enhancement of insulin output, in the islet:AEC co-cultures observed here supports the concept that soluble mediators exert positive paracrine influences on the islets maintained within the RCCS. Further, insulin-immunoreactivity persisted at the graft site of islet:AEC constructs beyond 30 days post-transplantation suggesting that local AEC-derived mediators may provide durable in vivo trophic support to $\beta$-cells.

The process of islet isolation inflicts a degree of damage on porcine islets, triggering secretion of specific inflammatory cytokines, including IL-1 $\beta$, IL-6 and TNF $\alpha$ which adversely impact $\beta$-cell function ${ }^{35,36}$. Additionally, the injury-induced production of proinflammatory molecules such as tissue factor and monocyte chemoattractant protein-1 by islets activates the innate immune response at the site of implantation, with subsequent loss of graft function ${ }^{34}$. Human AECs counter pro-inflammatory events in several models of inflammatory disease ${ }^{11,37,38}$, by attenuating proliferation of specific immune cell populations and inflammatory cytokine production within damaged tissues, whilst also orchestrating the transition of T-cells and monocytes from pro- to anti-inflammatory regulatory profiles ${ }^{39}$. Such functions would oppose the injurious impact of islet-derived pro-inflammatory molecules during RCCS co-culture and after transplantation.

Hyperacute rejection, mediated by alpha $(\alpha)$-Gal epitope, is a major concern in porcine solid organ xenotransplantation ${ }^{40}$ but the low expression or absence of $\alpha$-Gal on non-vascularised 
pig islets makes these an exception ${ }^{41,42}$. Instead, the primary effector cells underlying porcine islet xeno-rejection are considered to be CD4+ T-cells. This was confirmed with pig-tomouse islet xeno-transplants in which rapid ( $<7$ days) CD4+ T-cell-mediated $\beta$-cell rejection was dependent on antigen-presenting cells (APC) and attenuated by selective deletion of $\mathrm{CD} 4+\mathrm{T}$ cells, but not CD8+T cells ${ }^{22}$. Strategies which prevent the CD4+T-cell targeting of porcine islets are therefore likely to prevent xeno-rejection.

The present study investigates the proposition that co-culture with AEC alters the immune cell xeno-recognition of porcine islets by virtue of close spatial proximity. The mixed isletlymphocyte reaction (MILR) challenged both unmodified porcine islets and the robust cellular aggregates formed by their co-localisation with human AEC, showing that the heterotypic constructs inhibit lymphocyte proliferation. Potential soluble mediators underlying this response include TGF- $\beta$ released by AEC ${ }^{28}$ which in the context of the MILR would restrain $\mathrm{T}$-cell proliferation. The reduced $\mathrm{CD} 4+\mathrm{T}$-cell response with AEC-islet constructs in vitro also provides a possible explanation for the delay in rejection in immunecompetent mice. Although graft rejection was apparent in all treatment groups by the end of the study, AEC bearing islet grafts showed more persistent insulin immune-reactivity at $>30$ day post-transplant. Our morphological observations suggest that local AEC prolonged islet survival in the face of a fully operational recipient immune system, and increasing the ratio of AEC within the construct (viz. 1:1000) might potentiate the beneficial effect.

Further underlying the immune-protection of the AEC-bearing islets post-transplantation the AEC secretome includes IL-6 which alongside TGF- $\beta$ prevents dendritic cell (DC) maturation. This in turn enhances the secretion of IL-4 and IL-10 to evoke a tolerogenic microenvironment through suppression of helper T-cell proliferation and increased activity of 
T-regulatory cells (T-regs) ${ }^{43}$. AECs also express HLA-G, but exhibit low/absent level of expression of HLA class I and II molecules and an absence of costimulatory molecules CD80 (B7-1), CD86 (B7-2), CD40, or CD40 ligand ${ }^{44}$. AEC-secreted HLA-G mediates inhibition of T-cell proliferation and the suppression of the cytotoxic activity of Natural Killer (NK) cells 39 that may also have implications in relation to altered xenograft recognition and therefore for longer term islet immune-protection.

The moderate response of activated human $\mathrm{CD}+\mathrm{T}$-cells to unmodified islets was unexpected. Whilst biological variation may account for the non-significant numerical increase in CD4+ T-cells in the MILR assay, we have established that RCCS alone lowers the immunogenicity of human islets ${ }^{8}$, possibly by eliminating passenger leukocytes/ donor APCs, which in turn influence the ability of the CD4+ T-cells to mount a challenge against the tissue. Determining whether this also applies to porcine islets did not form part of the present investigation primarily as porcine islets maintained under conventional static culture (viz. seeded onto petri dishes) rapidly lose structural and functional viability. Thus static culture was not considered to be a reasonable control to the RCCS-maintained islet constructs. Yet, the concept has been previously reported ${ }^{45}$, and our observation that the immune-competent recipients rejected the non AEC-bearing islets at a slower tempo than reported elsewhere ${ }^{22}$ suggests that the period spent in RCCS may, itself, alter the xeno-reactivity of the graft material.

The use of bioreactor technology to co-localise cells across species barriers is novel and has important therapeutic implications. The limited availability of human cadaveric material ${ }^{46}$ is circumvented by use of porcine tissue, which is proposed to become a feasible source for human implantation following adoption of CRISPR-based gene editing to inactivate porcine endogenous retroviruses ${ }^{47}$. The incorporation of AEC to porcine islet grafts adds localised 
anti-inflammatory and immuno-modulatory properties that reduce xenogenicity. The benefit was illustrated by the sustainability of the islet-AEC constructs in a murine transplant model and highlights the translational potential of this approach. In the long-term it is possible that stem cell-modified xeno-islets may be applied clinically, possibly alongside the transient use of newly developed immunosuppressant drugs or other tolerogenic strategies ${ }^{48}$. By so doing, AEC-supplemented $\beta$-cell xeno-grafts may serve to enhance the accessibility and therapeutic outcome of islet transplantation. 


\section{REFERENCES}

1. Benhamou PY, Watt PC, Mullen Y, et al. Human islet isolation in 104 consecutive cases. Factors affecting isolation success. Transplantation. 1994;57(12):1804-1810.

2. Ricordi C, Gray DW, Hering BJ, et al. Islet isolation assessment in man and large animals. Acta Diabetol Lat. 1990;27(3):185-195.

3. Ricordi C, Tzakis AG, Carroll PB, et al. Human islet isolation and allotransplantation in 22 consecutive cases. Transplantation. 1992;53(2):407-414.

4. Abreu JR, Roep BO. Immune monitoring of islet and pancreas transplant recipients. Curr Diab Rep. 2013;13(5):704-712.

5. London NJ, Swift SM, Clayton HA. Isolation, culture and functional evaluation of islets of Langerhans. Diabetes Metab. 1998;24(3):200-207.

6. Kin T, Senior P, O'Gorman D, Richer B, Salam A, Shapiro AM. Risk factors for islet loss during culture prior to transplantation. Transpl Int. 2008;21(11):1029-1035.

7. Murray HE, Paget MB, Downing R. Preservation of glucose responsiveness in human islets maintained in a rotational cell culture system. Mol Cell Endocrinol. 2005;238(1-2):39-49.

8. Qureshi KM, Oliver RJ, Paget MB, Murray HE, Bailey CJ, Downing R. Human amniotic epithelial cells induce localized cell-mediated immune privilege in vitro: implications for pancreatic islet transplantation. Cell Transplant. 2011;20(4):523-534.

9. Qureshi KM, Lee J, Paget MB, et al. Low gravity rotational culture and the integration of immunomodulatory stem cells reduce human islet allo-reactivity. Clin Transplant. 2015;29(1):90-98.

10. Pischiutta F, Sammali E, Parolini O, Carswell HVO, Zanier ER. Placenta-Derived Cells for Acute Brain Injury. Cell Transplant. 2018;27(1):151-167. 
1

2

3

4

5

6

7

8

9

11. Alhomrani M, Correia J, Zavou M, et al. The Human Amnion Epithelial Cell Secretome Decreases Hepatic Fibrosis in Mice with Chronic Liver Fibrosis. Front Pharmacol. $2017 ; 8: 748$.

12. Burdette AJ, Guda T, Thompson ME, Banas R, Sheppard F. A Novel Secretome Biotherapeutic Influences Regeneration in Critical Size Bone Defects. J Craniofac Surg. 2018;29(1):116-123.

13. Zhu HT, Yu L, Lyu Y, Wang B. Optimal pig donor selection in islet xenotransplantation: current status and future perspectives. J Zhejiang Univ Sci B. 2014;15(8):681-691.

14. Buder B, Alexander M, Krishnan R, Chapman DW, Lakey JR. Encapsulated islet transplantation: strategies and clinical trials. Immune Netw. 2013;13(6):235-239.

15. Matsumoto S, Abalovich A, Wechsler C, Wynyard S, Elliott RB. Clinical Benefit of Islet Xenotransplantation for the Treatment of Type 1 Diabetes. EBioMedicine. 2016;12:255-262.

16. Morozov VA, Wynyard S, Matsumoto S, Abalovich A, Denner J, Elliott R. No PERV transmission during a clinical trial of pig islet cell transplantation. Virus research. $2017 ; 227: 34-40$

17. Paget M, Murray H, Bailey CJ, Downing R. Human islet isolation: semi-automated and manual methods. Diab Vasc Dis Res. 2007;4(1):7-12.

18. Murray HE, Paget MB, Bailey CJ, Downing R. Sustained insulin secretory response in human islets co-cultured with pancreatic duct-derived epithelial cells within a rotational cell culture system. Diabetologia. 2009;52(3):477-485.

19. Goss JA, Schock AP, Brunicardi FC, et al. Achievement of insulin independence in three consecutive type- 1 diabetic patients via pancreatic islet transplantation using islets isolated at a remote islet isolation center. Transplantation. 2002;74(12):1761-1766. 
20. Sottong PR, Rosebrock JA, Britz JA, Kramer TR. Measurement of T-lymphocyte responses in whole-blood cultures using newly synthesized DNA and ATP. Clin Diagn Lab Immunol. 2000;7(2):307-311.

21. Molano RD, Pileggi A, Berney T, et al. Long-term islet allograft survival in nonobese diabetic mice treated with tacrolimus, rapamycin, and anti-interleukin-2 antibody.

Transplantation. 2003;75(11):1812-1819.

22. Olack BJ, Jaramillo A, Benshoff ND, et al. Rejection of porcine islet xenografts mediated by CD4+ T cells activated through the indirect antigen recognition pathway.

Xenotransplantation. 2002;9(6):393-401.

23. Lalain S, Chaillous L, Gouin E, Sai P. Intensity and mechanisms of in vitro xenorecognition of adult pig pancreatic islet cells by CD4+ and CD8+ lymphocytes from type I diabetic or healthy subjects. Diabetologia. 1999;42(3):330-335.

24. Yamada K, Sachs DH, DerSimonian H. Human anti-porcine xenogeneic T cell response. Evidence for allelic specificity of mixed leukocyte reaction and for both direct and indirect pathways of recognition. J Immunol. 1995;155(11):5249-5256.

25. Matsumoto S, Noguchi H, Takita M, et al. ET-Kyoto ductal injection and density-adjusted purification combined with potent anti-inflammatory strategy facilitated single-donor islet transplantation: case reports. Transplant Proc. 2010;42(6):2159-2161.

26. Toso C, McCall M, Emamaullee J, et al. Liraglutide, a long-acting human glucagon-like peptide 1 analogue, improves human islet survival in culture. Transpl Int. 2010;23(3):259265.

27. Yang Z, Chen M, Ellett JD, Carter JD, Brayman KL, Nadler JL. Inflammatory blockade improves human pancreatic islet function and viability. Am J Transplant. 2005;5(3):475-483. 
1

2

3

4

5

6

7

8

9

28. Li H, Niederkorn JY, Neelam S, et al. Immunosuppressive factors secreted by human amniotic epithelial cells. Invest Ophthalmol Vis Sci. 2005;46(3):900-907.

29. Wolbank S, Peterbauer A, Fahrner M, et al. Dose-dependent immunomodulatory effect of human stem cells from amniotic membrane: a comparison with human mesenchymal stem cells from adipose tissue. Tissue Eng. 2007;13(6):1173-1183.

30. Parolini O, Alviano F, Bagnara GP, et al. Concise review: isolation and characterization of cells from human term placenta: outcome of the first international Workshop on Placenta Derived Stem Cells. Stem Cells. 2008;26(2):300-311.

31. Koizumi NJ, Inatomi TJ, Sotozono CJ, Fullwood NJ, Quantock AJ, Kinoshita S. Growth factor mRNA and protein in preserved human amniotic membrane. Curr Eye Res. $2000 ; 20(3): 173-177$.

32. Fiaschi-Taesch NM, Berman DM, Sicari BM, et al. Hepatocyte growth factor enhances engraftment and function of nonhuman primate islets. Diabetes. 2008;57(10):2745-2754.

33. Hanley S, Rosenberg L. Transforming growth factor beta is a critical regulator of adult human islet plasticity. Mol Endocrinol. 2007;21(6):1467-1477.

34. Movassat J, Beattie GM, Lopez AD, Portha B, Hayek A. Keratinocyte growth factor and betacell differentiation in human fetal pancreatic endocrine precursor cells. Diabetologia. $2003 ; 46(6): 822-829$.

35. Johansson U, Olsson A, Gabrielsson S, Nilsson B, Korsgren O. Inflammatory mediators expressed in human islets of Langerhans: implications for islet transplantation. Biochem Biophys Res Commun. 2003;308(3):474-479.

36. Kruger B, Yin N, Zhang N, et al. Islet-expressed TLR2 and TLR4 sense injury and mediate early graft failure after transplantation. Eur J Immunol. 2010;40(10):2914-2924. 
37. Manuelpillai U, Moodley Y, Borlongan CV, Parolini O. Amniotic membrane and amniotic cells: potential therapeutic tools to combat tissue inflammation and fibrosis? Placenta. 2011;32 Suppl 4:S320-325.

38. Murphy S, Lim R, Dickinson H, et al. Human amnion epithelial cells prevent bleomycininduced lung injury and preserve lung function. Cell Transplant. 2011;20(6):909-923.

39. Magatti M, Vertua E, Cargnoni A, Silini A, Parolini O. The Immunomodulatory Properties of Amniotic Cells: The Two Sides of the Coin. Cell Transplant. 2018;27(1):31-44.

40. Vaughan HA, Loveland BE, Sandrin MS. Gal alpha(1,3)Gal is the major xenoepitope expressed on pig endothelial cells recognized by naturally occurring cytotoxic human antibodies. Transplantation. 1994;58(8):879-882.

41. McKenzie IF, Koulmanda M, Mandel TE, Sandrin MS. Pig islet xenografts are susceptible to "anti-pig" but not Gal alpha(1,3)Gal antibody plus complement in Gal o/o mice. J Immunol. 1998;161(10):5116-5119.

42. Heald KA, Carless N, Jay TR, Boucher N, Downing R. Expression of the GALalpha(13)GAL epitope on pig islets. $J$ Mol Med (Berl). 1999;77(1):169-171.

43. Silini AR, Magatti M, Cargnoni A, Parolini O. Is Immune Modulation the Mechanism Underlying the Beneficial Effects of Amniotic Cells and Their Derivatives in Regenerative Medicine? Cell Transplant. 2017;26(4):531-539.

44. Caruso M, Evangelista M, Parolini O. Human term placental cells: phenotype, properties and new avenues in regenerative medicine. Int J Mol Cell Med. 2012;1(2):64-74.

45. Lacy PE, Finke EH, Janney CG, Davie JM. Prolongation of islet xenograft survival by in vitro culture of rat megaislets in 95\% O2. Transplantation. 1982;33(6):588-592. 
46. Ausania F, Drage M, Manas D, Callaghan CJ. A registry analysis of damage to the deceased donor pancreas during procurement. Am J Transplant. 2015;15(11):2955-2962.

47. Niu D, Wei HJ, Lin L, et al. Inactivation of porcine endogenous retrovirus in pigs using CRISPR-Cas9. Science. 2017;357(6357):1303-1307.

48. Chhabra P, Brayman KL. Current status of immunomodulatory and cellular therapies in preclinical and clinical islet transplantation. J Transplant. 2011;2011:637692. 


\section{FIGURE LEGENDS}

\section{Figure 1}

Porcine islets imaged (A) immediately following isolation and (B) following 48 hours in RCCS (magnification $\mathrm{x} 20$ ). Fragmented porcine islets reassembled in rotational culture to form stable cell aggregates of 150-200um diameter. Immuno-staining of $(C, F)$ porcine insulin and $(D, G)$ human CK19 following a 48 hour period of co-culture within the RCCS. The two cell types integrated to form tight heterotypic constructs with AEC interacting with cells at the periphery of the islet $(\mathrm{E}, \mathrm{H})$. Fluorescence indicates presence of insulin (TRITC) or CK 19 (FITC). Magnification x100 (C-H).

\section{Figure 2}

Upper panel: Insulin-secretory capacity of porcine islets following 48 hours of RCCS culture either alone or in combination with AEC: Islets incubated in the presence of basal glucose $(1.67 \mathrm{mmol} / \mathrm{l}$; open bars), high glucose and arginine (glucose $-16.7 \mathrm{mmol} / \mathrm{l}$, arginine $10.0 \mathrm{mmol} / \mathrm{L}$, shaded bars) or high glucose, arginine + theophylline (theophylline - 10mmol/l; closed bars). Results represent the mean S.I. \pm s.e.m. $n=4$ independent observations. ${ }^{*} \mathrm{p}<0.05$, for stimulated insulin secretion from islet:AEC constructs vs. control (basal secretion); $\uparrow \mathrm{p}<0.01$ for stimulated insulin release from constructs formed from porcine islets co-cultured with AEC at a ratio of 1:1000 vs. AEC ratio of $1: 100$.

Lower Panel: Xeno-response of activated human CD4+ T-cell subsets (CD4(ac)) to porcine islets and islet:AEC constructs. Unmodified islets or constructs formed from porcine islet clusters associated with human AEC in RCCS for 48 hours were subjected to MILR assay. T-cell proliferation in response to anti-CD3/CD28, unmodified islets or islet:AEC constructs were expressed as percentage of the control group $(\mathrm{CD} 4+(\mathrm{rs}))$. Results represent the mean \pm s.e.m. $n=4$ observations, 4 replicates per group. $* \mathrm{p}<0.05$ for T-cell expansion in response to AEC-bearing constructs at the two ratios tested vs. unmodified islets. 


\section{Figure 3}

H\&E images of the graft site (A, C, E - magnification x20) and immunohistochemical staining for insulin (red, B, D, F - magnification x 100) and CK19 (F, green) viewed in sections of explanted liver 14 days post-transplantation. In immunosuppressed recipients of unmodified islets $(n=3)$ a clear area of graft material could by seen embedded within the liver parenchyma (*) with H\&E staining (A). In B the largest area of staining observed (bordered area) is viewed following immunostaining with anti-porcine insulin antibody indicating the presence of islets. Immune-competent recipients display reduced cell density at the presumptive graft site (C -bordered area) and low levels of insulin immunoreactivity (D). Islet:AEC constructs transplanted into immune competent recipients $(n=3)$ displayed preserved insulin and CK19 immunoreactivity 14 days post-transplant.

\section{Figure 4}

H\&E images of the graft site (A, C, E - magnification x20) and immunohistochemical staining for insulin and CK19 (insulin - red, CK19 -green, B, D, F- magnification x 100) viewed in sections of explanted kidney at 7 (A,B), 14 (C,D) and >30 (E,F) days post-transplantation. All recipients received islet:AEC grafts with no systemic immunosuppression (n=3). At 7 (A) and 14 (C) days postimplantation, insulin-positive cells were found in close proximity to AEC. At $>30$ days fewer insulin or CK19 immunoreactive cells were evident at the graft site, although the tempo of rejection was delayed compared to immune competent mice receiving unmodified islets at this time point. 


\section{ACKNOWLEDGEMENTS}

We gratefully acknowledge the financial support of The Rowlands Trust, The Eveson

Charitable Trust, The James Tudor Foundation, The Sir Halley Stewart Trust, The South

Warwickshire Diabetes UK Voluntary Group and Worcestershire Acute Hospitals NHS Trust R\&D.

\section{CONFLICT OF INTEREST}

The authors declare that there is no conflict of interest.

\section{AUTHOR CONTRIBUTION}

AZ, JL, HEM and RD contributed to the conception and design of the study. AZ, JL, SY, MBP, CJB, HEM and RD performed experiments and analysed data. AZ, CJB, HEM and RD contributed to data analysis/interpretation. All authors contributed to the drafting/revision and final approval of the manuscript. 
Figure 1
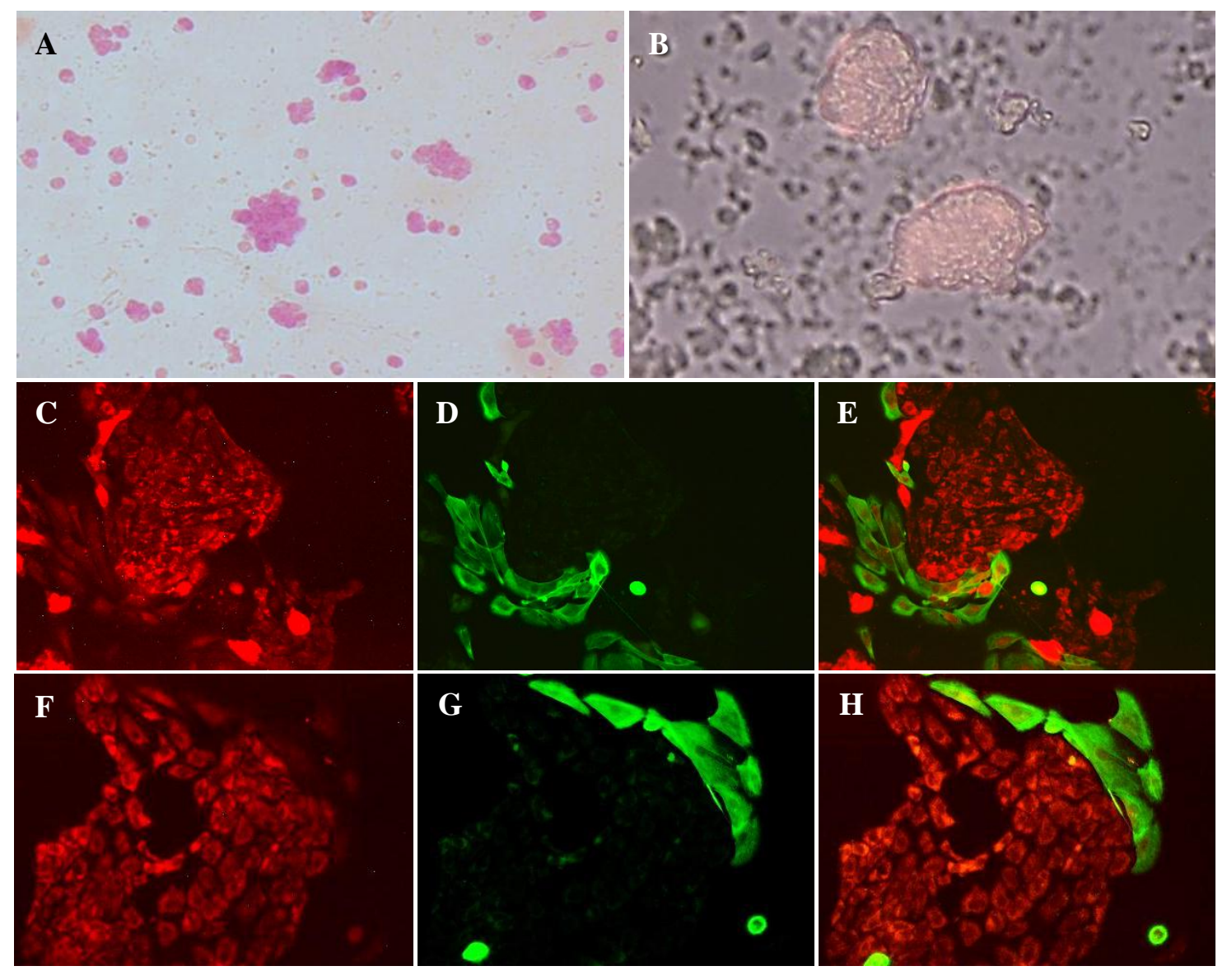
Figure 2
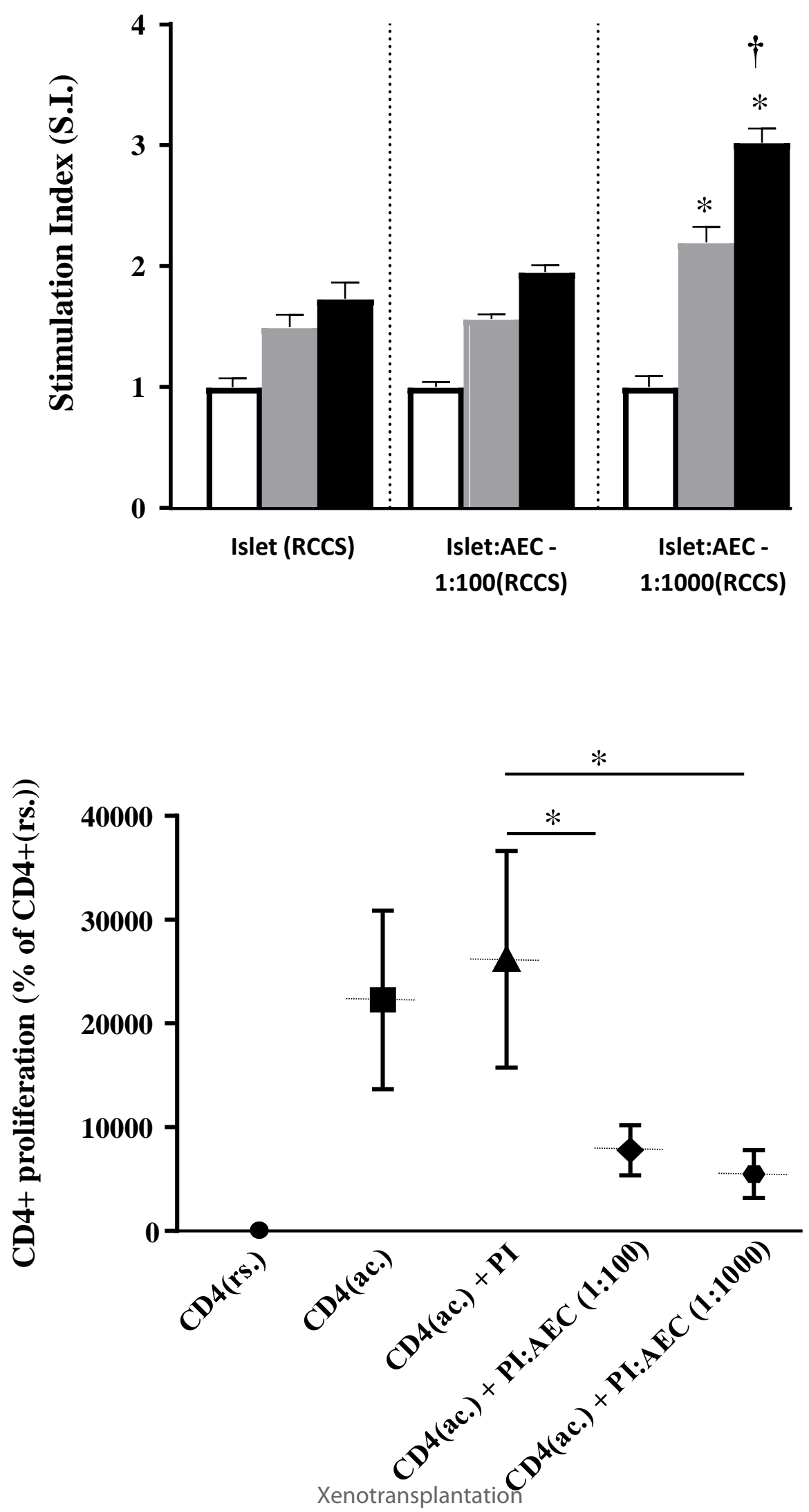
Figure 3
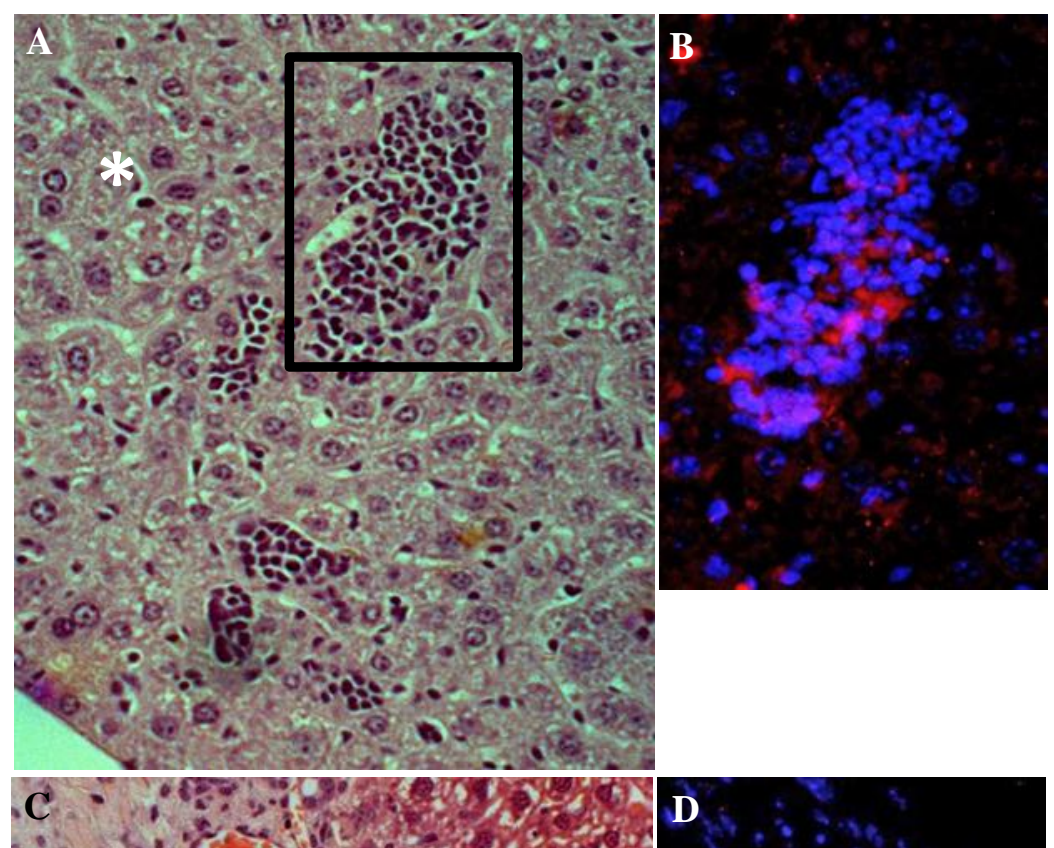

D
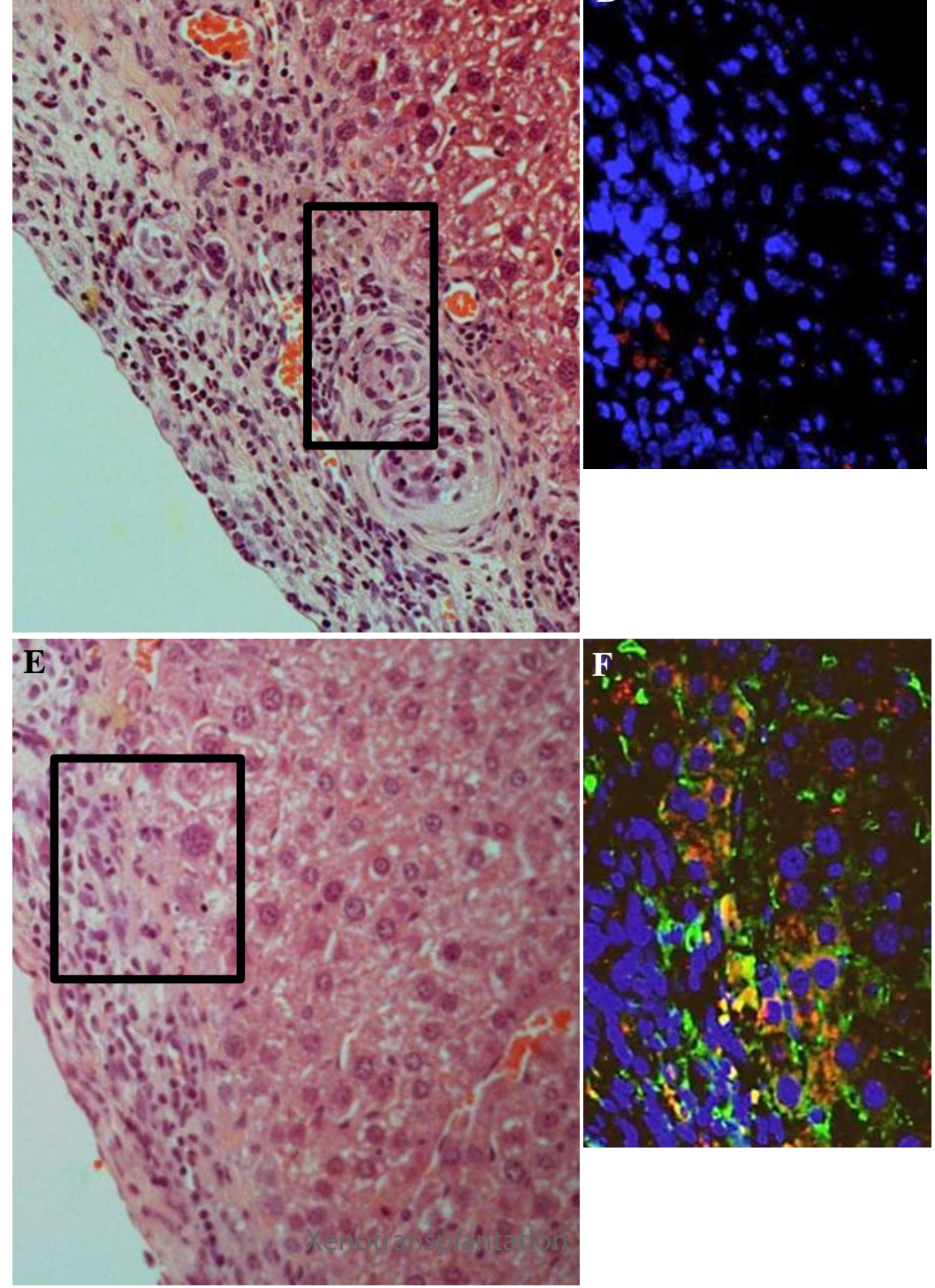
